\title{
S5. Problems with translations in psychiatry
}

\section{PROBLEMS IN TRANSLATIONS IN PYCHIATRY - GENERAL PROBLEMS \\ N. Sartorius}

Department of Psychiatry, University of Geneva, Switzerland

Techniques for the production of equivalent versions of instruments used for the assessment of factors relevant to psychiatry (e.g., of mental states, psychiatric history, personality traits) have been significantly improved over the past two decades. They are more widely used and the awareness of the importance of their application has grown. Methodological problems remain, however. The areas in which further methodological development is necessary include the application of verbal and nonverbal tests for the assessment of cognitive difficulty and impairment; comparisons of results obtained by self-report questionnaires in which comments or replies of the subjects are not recorded; and questionnaires in which the order of questioning and foreseeable answers ae highly standardized.

The paper will discuss some of these problems and a translation procedure developed and tested in several major international collaborative studies coordinated by the World Health Organization.
PROBLEMS WITH TRANSLATION FROM ENGLISH TO FRENCH JD Guelfi* P Rousseau-Cunningham** *Editor in chief, European Psychiatry, 100 rue de la Santé, Paris 75674 , * European Psychiatry, Consulting Translator

Several reviews of procedures used in translating instruments to asses psychiatric symptoms, behavioural features, or personality traits have been published recently (Sartorius, 1979, 1994).

Some differences in meaning are difficult to detect and can distort the results (Prince and Mombour, 1967).

The use of bilingual translators and focus groups helps to identify terms for which semantic equivalence should be carefully examined. The two other types of equivalence are the conceptual equivalence (e.g. the different use of the conditional tense in English-speaking countries and in France). Some examples of these problems will be given according to our personal experiences of the French translation of several american or English instruments = DSM-III in 1982-1983, DSM-III-R in 1988-1989, ICD-10 from 1990 to 1994 (coordinator: Pull) MMPI-2 (with the collaboration of Simon and Giller from the CPA-Centre de Psychologie Appliquée) and some recent studies on several Quality of Life Scales such as the General WellBeing Scale (Dupuy, 1974) or the Bradley Well-Being Scale.

The best translation procedure is obtained by applying the two versions to bilingual groups of investigators. Other methods are less time-consuming but the reiterative back translation is a necessary step for fully standardized instruments. 


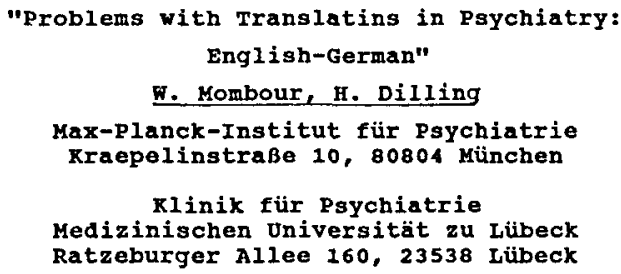

The paper describes some general problems of translating diagnostic texts from English to German. Besides ICD-10 and DSM-III-R several clinical interviews such as SCAN and CIDI have been translated and the German translations have to correspond to each other. Although the German and English languages are closely related, certain problems were found if the words for instance resembled each other but their meanings were very different. Therefore the translator will encounter many potential traps, even if the language seems to be very familiar and texts easy to translate. Some examples are given in which the German translation was mistaken at first glance, some others in which in German appropriate expressions were not found. Some English diagnostic terms have been used for many years, so that a translation into German would not have been accepted by the users. In summary the translations have proved to be much more difficult than foreseen.

\section{PROBLEMS WITH TRANSLATIONS IN PSYCHIATRY: ENGLISH - SPANISH \\ JJ López-Ibor, Jr.}

Psychiatric Department. Complutense University. Martín Lagos s/n. 28035, Madrid, Spain.

Spanish psychiatric tradition is one of eclecticism with strong influence both German and French tradition. In the last decades the angloamerican influence has been important and usually well integrated. For instance, the DSM-III Classification and Nomenclature has been extremely well accepted al over the country. ICD-10 brought a new challenge as it was developed simultaneously in several languages among them English and Spanish. During the preparation meetings of ICD-10 the author of this presentation often presented his views thinking in how a document would be written in Spanish. This lead on the whole to prevent last minute translation problems. On the other hand ICD-9 Spanish version was produced in an extremely correct language while the DSM-III translation was full of americanisms some of them well accepted in psychiatric practice. The researchers involved in field trials gave also advice on the terminology. Several terms required a more in depth consideration such as disorder, delusion, affect (mood), anxiety and pervasive. In spite of all this the modern trend to avoid the traditional greek etimology in order to overcome the vagueness of past concepts has lead to the use of a too artificial language. The term "trastorno de ideas delirantes persistentes" to avoid "paranoia" in the sake of exactness, is too long for every day use. The author would like to recover old terminology once new classifications have made the concepts more precise.
PROBLEMS WITH TRANSLATIONS IN PSYCHIATRY: ENGLISH - $\mathrm{CZECH}$

P. Smolik

Postgraduate Medical Institute, Prague, Czech Republic

Four basic groups of problems have appeared regularly during the work at English - Czech translations of psychiatric texts:

1. Etymological questions.

Examples: somatoform versus somatomorph psychoactive versus psychactive

2. Semantic questions and changes of routine accustomed terms.

Examples: episode versus phase, attack

3. Adoption of new terms. Alternatives of translation:

a) use of the original czech word;

b) adoption of the original English word in czech grammatical form;

c) adoption of another foreign word, which is well accustomed and has adequate sense;

Examples: flashback, sleepwalking disorder.

4. The symbolic - phonetic balance.

Example: "Spell WORLD backwards."

Which of czech words have similar symbolic sense and phonological structure like WORLD?

\section{PROBLEMS WITH ENGLISH-POLISH TRANSLATIONS IN PSYCHIATRY}

St. Pużý́ski, J. C. Czabala

Institute of Psychiatry and Neurology, Sobieskiego 1/9, 02-957 Warsaw, Poland.

The authors begin their paper by pointing to the traditional bond between Polish and Western European psychiatry. The concepts of both $\mathrm{E}$. Kraepelin and $\mathrm{E}$. Bleuler - from the very beginning known in Poland - considerably influenced theoretical views of Polish psychiatrists and their classification of mental disorders before World War II. The bonds with Western European psychiatry were not broken by the war. After a few years' interval (1948-1956) Polish medical schools and research centers have renewed vivid contacts with their counterparts in Western Europe and the USA. Not only major approaches in psychiatry, but also the accepted in the West classifiaction systems, diagnostic criteria of research tools (scales and inventories) for assessment of psychopathology are well known in Poland; some of them are very popular. Scientific journals (in English mostly) and personal contacts are the main sources of information. Unfortunately, the number of manuals and monographs translated into Polish is hardly sufficient (since World War II, i.e. during almost 50 years, only books have been translated). Analyzing the causes of this shortage, the authors discuss both subjective and objective difficulties, e.g. the state monopoly for publishing medical books, a very long publishing process, difficulties with obtaining copyrights, etc. A noticeable movement in the publishing market has appeared in recent years; there are more and more small publishing firms, and quite a number of English language publications in psychiatry are to be translated and published in Poland. Finally, terminological problems and difficulties in the translation of English psychiatric texts into Polish and vice versa are discussed. 
WHO CROSS-CULTURAL APPLICABILITY RESEARCH (CAR)

STUDY

A Janca*, LA Bennett

"Division of Mental Health, World Health Organization, 1211 Geneva 27, Switzerland

This WHO project examined the cross-cultural applicability of diagnostic criteria and instruments for the assessment of substance use disorders. Nine centres representing different cultures and a wide range of languages participated in this study. One of the components of the study was related to problems with the translation of concepts, criteria and items in the instruments. A bilingual expert consultation group was established at each centre to assist in translation and back-translation of the instruments. In addition to the bilingual expert groups, monolingual groups were also involved in the assessment of the comprehensibility of the translations. These monolingual groups were composed of people who were likely to represent the general population to be drawn upon in the study and who spoke only the native language. Translation and backtranslation procedures and the work of the bilingual and monolingual expert groups are described in this paper. 